\title{
Comparing endoscopic ultrasound (EUS)- guided fine needle aspiration (FNA) versus fine needle biopsy (FNB) in the diagnosis of solid lesions: study protocol for a randomized controlled trial
}

\author{
Jinlin Wang ${ }^{1}$, Xiaoli Wu' ${ }^{1}$, Ping Yin², Qiaozhen Guo ${ }^{1}$, Wei Hou' ${ }^{1}$ Yawen $\mathrm{Li}^{1}$, Yun Wang ${ }^{1}$ and Bin Cheng ${ }^{1 *}$
}

\begin{abstract}
Background: Linear endoscopic ultrasonography (EUS) allows the visualization, identification, and characterization of the extent of lesions of the gastrointestinal (GI) tract and adjacent structures. EUS-guided fine-needle aspiration (EUS-FNA) facilitates a more accurate diagnosis of mediastinal, intra-abdominal, and pancreatic lesions through the collection of the cytological material under direct visualization. Recent reports suggest that histological samples can be obtained by EUS-FNA with a reverse, bevel-tipped needle (the ProCore needle) to collect the core samples (fine needle biopsy, FNB), thereby adding a new dimension to the diagnostic usefulness of this technique. Certain neoplasms, such as lymphoma and stromal tumors, can be assessed by EUS-FNB to confirm the diagnosis. Here, we aimed to carry out a prospective, multicenter, single-blind, randomized, controlled trial to compare EUS-FNB and EUS-FNA.
\end{abstract}

Methods/design: A total of 408 patients will be enrolled from five endoscopic centers. Patients will be divided into two groups: (1) group A, which is the EUS regular needle group (EUS-FNA) and (2) group B, which is the EUS ProCore needle group (EUS-FNB). Patients in group A will be examined with a 22G EchoTip Ultra needle, and patients in group B, with a 22G EchoTip ProCore needle. For all included patients, four EUS-guided passes will be made in each lesion. In the first and second pass, a slow-pull suction method of the stylet will be done. The third and fourth pass will use manual suction of $5 \mathrm{cc}$. The primary objective is to compare the diagnostic yield of malignancy by EUS-FNA versus EUS-FNB.

Discussion: The trial will compare samples obtained by EUS-FNA versus EUS-FNB for the diagnostic yield of solid lesions. The efficacy of these two sampling methods will be assessed on various lesions, which may provide insights into developing practice guidelines for their future indications.

Trial registration: Clinical Trials.gov, NCT02327065.

Keywords: EUS-FNA, EUS-FNB, Slow-pull, Suction, Solid lesions, Diagnostic yield

\footnotetext{
* Correspondence: b.cheng@tjh.tjmu.edu.cn

${ }^{1}$ Department of Gastroenterology and Hepatology, Tongji Hospital, Tongji

Medical College, Huazhong University of Science and Technology, Wuhan

430030, China

Full list of author information is available at the end of the article
}

\section{$\int$ Biomed Central}

(c) 2016 Wang et al. Open Access This article is distributed under the terms of the Creative Commons Attribution 4.0 International License (http://creativecommons.org/licenses/by/4.0/), which permits unrestricted use, distribution, and reproduction in any medium, provided you give appropriate credit to the original author(s) and the source, provide a link to the Creative Commons license, and indicate if changes were made. The Creative Commons Public Domain Dedication waiver (http://creativecommons.org/publicdomain/zero/1.0/) applies to the data made available in this article, unless otherwise stated. 


\section{Background}

Linear endoscopic ultrasonography (EUS) allows the identification of suspected malignancies by creating real-time images of the digestive tract and adjacent lesions [1]. EUS-guided fine needle aspiration (FNA) offers an opportunity for sampling mediastinal, intraabdominal, and pancreatic lesions under direct visualization [2]. Since its original description in the early 1990s, EUS-FNA has been used to obtain cytological material, thereby contributing to the prompt and accurate diagnosis of clinically suspected lesions [3-6]. The diagnostic accuracy of EUS-FNA is generally high, from $52 \%$ to $94 \%$ [7-10]. Cytopathology plays an important role in improving the diagnostic yield. Notably, distinguishing the inflammatory lesion caused by a reaction and regeneration from welldifferentiated neoplasia solely based on cytological evaluation can be difficult. Moreover, certain neoplasms, such as lymphoma and stromal tumors, require histological exams to assess the tissue architecture and cell morphology changes in order to confirm the diagnosis [11]. To overcome this limitation, a new fine needle biopsy (FNB) device has been designed (Cook Endoscopy, Limerick, Ireland). The new ProCore needle was designed with a reverse bevel at the tip to collect a core sample. A multicenter study on 114 patients showed that the application of the ProCore needle (EUS-FNB) led to $85.96 \%$ diagnostic accuracy; $90.2 \%$ sensitivity to malignancy, $99 \%$ specificity, $100 \%$ positive predictive value (PPV), $78.9 \%$ negative predictive value (NPV), and $92.9 \%$ accuracy [11]. Overall, most studies favored EUS-FNB for achieving an adequate histological specimen and high diagnostic yield compared to EUS-FNA [11-16]. However, several studies have suggested that the diagnostic yields of EUS-FNB and EUS-FNA were similar [17-19]. Therefore, the impact of the needle type (ProCore or regular) on the diagnostic yield needs to be further studied. We plan to carry out a randomized controlled trial to compare the diagnostic yield of EUS-FNA versus EUS-FNB and to assess the sample quality obtained by both the slow-pull and the suction approaches.

\section{Methods/design}

The study was approved by The Ethics Committee of Tongji Medical College, Huazhong University of Science and Technology (HUST) (IORG No: IORG0003571). Informed consent will be obtained from the patients or from their closest relatives with authorization.

\section{Study design}

A total of 408 patients from five endoscopic centers in China will be enrolled in this prospective, multicenter, randomized, controlled trial. They will be randomly divided into two groups: (1) group $\mathrm{A}$, which will receive the EUS procedure using the regular needle (EUS-FNA, 22G EchoTip Ultra needle, Cook Endoscopy, Limerick, Ireland) and (2) group $B$, which will receive the EUS procedure using the ProCore needle (EUS-FNB, 22G EchoTip ProCore needle, Cook Endoscopy, Limerick, Ireland). The recruitment process flowchart is shown in Fig. 1.

\section{Objectives}

The primary objective of this study is to compare EUS-FNA versus EUS-FNB for the diagnostic yield (\%) of malignancy [13]. The secondary objectives are to compare EUS-FNA versus EUS-FNB for 1) the overall diagnostic yield (\%) from solid lesions; 2) the diagnostic yield (\%) from pancreatic and nonpancreatic solid lesions, 3) the crossover diagnostic yield (\%), and 4) the sample quality obtained by either the slow-pull or suction approaches, respectively.

\section{Setting}

A total of 408 patients will be recruited from five endoscopic centers, including Tongji Hospital, Tongji Medical College, HUST, Wuhan; Cancer Institute \& Hospital, Chinese Academy of Medical Sciences, Peking Union Medical College, Beijing; Fudan University Shanghai Cancer Center, Shanghai Medical College, Fudan University, Shanghai; Sun Yat-sen University Cancer Center, Guangzhou; and the First Affiliated Hospital, College of Medicine, Zhejiang University, Hangzhou. All clinic sites meet the following criteria: (1) at least 48 patients can be recruited per site; (2) at least 2 to 4 trained physician(s) and nurse practitioner(s) per center can participate in the trial; and 3) the site agrees to strictly abide by the study protocol.

\section{Eligibility \\ Inclusion criteria}

Patients who meet the following inclusion criteria will be recruited to the trial:

1) Age $>18$ years old

2) Sex: male or female

3) Patients who require endoscopic ultrasound and tissue sampling after imaging examination (MRI, CT, and ultrasonography) that shows either pancreatic, intra-abdominal, mediastinal, or pelvic cavity solid lesions $($ size $>1 \mathrm{~cm}$ )

4) Patients who are willing to be examined in the trial centers

5) Patients who are able to give consent

\section{Exclusion criteria}

Patients will be excluded from the trial for any of the following reasons: 


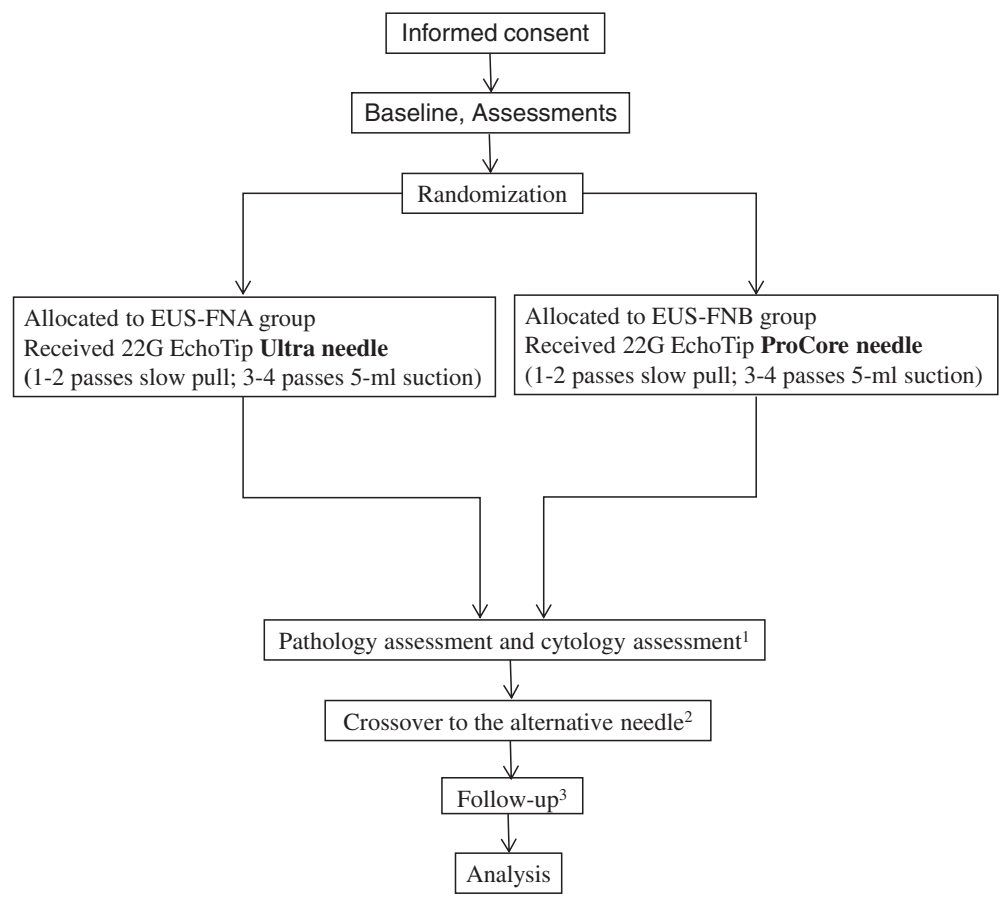

Fig. 1 CONSORT flowchart illustrating the randomization and recruitment process in the study. ${ }^{~}$ Each slide will be assessed by two independent experts. The cytologists and pathologists will follow the protocol to assess the samples and will be blinded as to which technique is used for which specimen. ${ }^{2}$ If diagnostic failure occurs, the patient will be allowed to crossover to the other group after agreeing to accept a needle biopsy again on the same lesion 1 week later. ${ }^{3}$ Three follow-up points are scheduled after the biopsy (1-week postoperational follow-up, 12-week follow-up, and 48-week follow-up); thereafter, the follow-ups will be conducted via telephone interviews or outpatient interviews. Once the surgical pathologic results are obtained, we will stop the follow-up

1) Hemoglobin $\leq 8.0 \mathrm{~g} / \mathrm{dL}$

2) Pregnant female

3) Patient has any coagulation disorder (PLT $<50,000 /$ $\mathrm{mm}^{3}$, INR $>1.5$ )

4) History of taking oral anticoagulation agents such as aspirin, warfarin, etc. in past week

5) Experienced acute pancreatitis in past 2 weeks

6) Has cardiorespiratory dysfunction that cannot tolerate the operation

7) Has mental diseases or drug addiction

8) Unable to provide informed consent

\section{Randomization and blinding}

The randomization will be conducted at the study office at the School of Public Health, Tongji Medical College, HUST. A stratified block randomization will be used, and the block size is 8 . The patients will be allocated randomly to either group A (EUS-FNA) or group B (EUS-FNB) (1:1).

A data manager, who will not be involved in the data analysis or patient enrollment, will generate the randomization schedule. To ensure allocation concealment, the randomization schedule will be sealed under scratch cards (001-408). The scratch cards will not be opened until the baseline assessment is completed and the patient has consented to participate in the study.

The randomization schedule will not be available to the study recruiters or echoendoscopists until the baseline assessment is completed. Only the study coordinators have access to the randomization schedule. The cytologists and pathologists will be blinded during the entire study.

\section{Interventions}

Patients will be allocated randomly into group A (EUS-FNA, $\mathrm{n}=204$ ) or group B (EUS-FNB, $\mathrm{n}=204$ ). Group A patients will be examined with the 22G EchoTip Ultra needle, and group $B$ will be examined with the 22G EchoTip ProCore needle.

The procedure will be performed under deep sedation according to the principles of "monitored anesthesia care." The patients will be anesthetized with intravenous administration of propofol by trained anesthetists. All patients will receive oxygen during the procedures; blood pressure and heart rate will be monitored. Once the lesion is evaluated by EUS, the echoendoscopist will select the shortest pathway, while avoiding blood vessels, to reach the lesion. Under real-time visualization, each lesion will be punctured with four needle passes. To 
eliminate technical biases, the same procedure for obtaining samples will be applied to each patient undergoing EUS-FNA or EUS-FNB.

\section{Technique for EUS-FNA and EUS-FNB}

1) The first and second needle passes (slow pull) [20]: The needle will be advanced into the lesion under realtime EUS visualization. Back and forth movements will be made 20 times within the lesion and will be performed using simultaneous minimal negative pressure by pulling the needle stylet slowly and continuously.

2) The third and fourth needle passes (5-ml suction) [18]: After the needle has been advanced into the lesion under real-time EUS visualization, the stylet will be removed. Continuous suction will be applied with a 5 -ml syringe, and the needle will be moved back and forth 20 times within the lesion. Suction will be released, and then, the needle will be withdrawn from the lesion.

After biopsy, the samples will be labeled immediately with numbers in the order of the needle pass. Samples will be prepared for histological and cytological examinations. The cytologists and pathologists will always be blinded regarding which technique was used for which specimen.

After the selected lesion has been punctured during the four needle passes, if no core tissue is obtained or if the operators determine the specimens are insufficient according to the results of the macroscopic onsite evaluation (MOSE) [21], the operators will use a proper puncture method to obtain samples as the backup procedure.

If diagnostic failure occurs, the patient will crossover to the other arm after agreeing to accept a needle biopsy again on the same lesion 1 week later with the same method mentioned above.

\section{Assessment standards}

After patient recruitment is completed, all samples will be reassessed by selected cytologists and pathologists from Tongji Hospital, Tongji Medical College, HUST, and from the Cancer Institute \& Hospital, Chinese Academy of Medical Sciences, Peking Union Medical College. They will assess the samples following the protocol and remain blinded regarding which technique was used for which specimen. Each sample will be assessed by two independent experts. If the two experts have different judgments, they should discuss it together and make a final decision [7].

\section{Histology assessment criteria}

The histological assessment will involve the following:

1) Tissue integrity assessment $[20,22]$

Grade A: core tissue (an architecturally intact piece of tissue measuring at least 550 micron in greatest axis, as the diameter of a high-power microscopic field), clearly characterizes the lesion sufficient for diagnosis

Grade B: core fragments present, tissue does not meet the criteria for architecturally intact histology but can still yield a diagnosis based on cell morphology

Grade C: no lesion tissue found and cannot yield a diagnosis

2) Blood cell contamination assessment [18]

Grade A: little blood contamination, minimal

surface area $(\mathrm{SA})<25 \%$ of slide

Grade B: medium blood contamination, 25-50 \% of the slide

Grade C: much blood contamination, SA $>50 \%$ of the slide

\section{Cytology assessment criteria}

The cytological assessment involves the following:

1) Cellularity assessment [9]

Grade A: satisfactory, more than four clusters, with a minimum of ten cells in each cluster Grade B: adequate, approximately two to four clusters, with a minimum of ten cells in each cluster

Grade C: unsatisfactory, fewer than two clusters, or no cellular smear

2) Blood cell contamination assessment [18] Grade A: little blood contamination, SA $<25 \%$ of slide

Grade B: medium blood contamination, 25-50 \% of slide

Grade C: much blood contamination, SA > $50 \%$ of the slide

\section{Collecting data}

Table 1 shows the types of data collected and when the data should be collected.

\section{Final diagnosis}

The reports will be stratified into four diagnostic categories for the histological and cytological evaluation, including "positive for malignancy," "suspicious for malignancy," "atypia," and "negative for malignancy." The "positive for malignancy" will be considered if the reports contain words such as "diagnostic for malignancy," "compatible with carcinoma," "consistent with adenocarcinoma," "positive for malignant cells," "malignant cells present," or when specified by the exact tumor type [23]. The "positive for malignancy" will not be considered if the reports contain words such as "suspicious for malignancy," "atypia," or "negative for malignancy." 
Table 1 Study schedule for data collection

\begin{tabular}{|c|c|c|c|c|}
\hline \multirow[t]{2}{*}{ Item } & Interview 1 & Interview 2 & Interview 3 & Interview 4 \\
\hline & 1-0 week before EUS & 0-1 week after EUS & 12 weeks \pm 5 days after EUS & 48 weeks \pm 5 days after EUS \\
\hline Informed content & $x$ & & & \\
\hline Inclusion criteria & $x$ & & & \\
\hline Patient characteristics & $x$ & & & \\
\hline Blood routine tests & $x$ & $x$ & & \\
\hline Coagulation routine tests $^{1}$ & $x$ & & & \\
\hline Blood biochemistry tests ${ }^{2}$ & $x$ & $x$ & & \\
\hline Blood tumor markers tests ${ }^{3}$ & $x$ & & $x$ & $x$ \\
\hline Complications & & $x$ & $x$ & $x$ \\
\hline Clinical signs ${ }^{4}$ & $x$ & $x$ & $x$ & $x$ \\
\hline Imaging examination & $x$ & $x$ & $x$ & $x$ \\
\hline Cytology examination & & $x$ & & $x$ \\
\hline Pathology examination & & $x$ & & $x$ \\
\hline Surgical-pathological examination & & $x$ & $x$ & $x$ \\
\hline Therapies & & X & $x$ & X \\
\hline
\end{tabular}

${ }^{1}$ Coagulation routine tests: prothrombin time (PT), activated partial thromboplastin time (APTT), thrombin time (TT), fibrinogen, INR

${ }^{2}$ Blood biochemistry tests: AST, ALT, BUN, Cr, ALP, lipase, amylase

${ }^{3}$ Blood tumor marker tests: CEA, CA19-9, CA72-4, AFP, SSC, NSE

${ }^{4}$ Clinical signs include pain, weight loss, cachexia, etc.

\section{Final diagnostic criteria}

The final diagnostic criteria [20] include the following:

1) For patients who undergo surgery, the final diagnoses will be based on the definite histological diagnoses from surgical resection specimens.

2) In the absence of surgical pathology, a minimum 48 weeks of clinical follow-up time will be conducted. If the lesion spontaneously resolves or has no sign of deterioration in follow-up imaging studies, the lesion will be considered a benign disease. If the lesion shows enlargement or metastasis, and the patient presents malignant symptoms such as weight loss, anemia, or dies of tumor complications during the follow-up, the lesion will be considered a malignant disease.

\section{Statistical analysis}

\section{Sample size}

We assumed that the diagnostic yield of malignancy for EUS-FNA was $80 \%$ [23] versus $93 \%$ for EUSFNB $[11,13]$. Because approximately $70 \%$ of patients are expected to be diagnosed with malignancy [9], the sample size should be 342 , with a power of $85 \%$ and a two-sided significance level of $5 \%$. Considering $20 \%$ of patients will be lost to follow-up, the estimated patient number in this trial should be 408 .

\section{Data analysis}

A two-tailed distribution will be used and a $P$-value $<0.05$ is considered statistically significant. Continuous variables will be measured as mean (range) and standard deviation using $t$ tests or Wilcoxon rank-sum tests. Categorical variables will be measured as the count and percentage using the $x^{2}$ test. The diagnostic yield will be described as a proportion using the $x^{2}$ test; the blood contamination and cellularity in specimens with slow-pull and suction will be divided into three levels (Grade A, Grade B, and Grade C), and McNemar's test for correlated proportions will be used. All analyses will be performed with SAS version 9.2.

\section{Discussion}

The role of EUS-FNA in obtaining cytological materials is well-established [24]. However, a recent study suggests the false positive rate of FNA cytology is $5 \%$ to $7 \%$, which is higher than the originally reported rates of $0 \%$ to $1 \%[25,26]$. Moreover, an increased interest in histological tissue sampling under real-time EUS imaging exists because of the advantage in diagnosing certain suspected tumor types, such as neuroendocrine tumor and lymphomas [27]. A standard 19G FNA needle has been used to obtain histological materials and achieved a diagnostic accuracy of $94.5 \%$ [28], although the device is highly associated with technical failures. A Quick-Core needle was used with an overall diagnostic accuracy of $75 \%$ to $84 \%[29,30]$. One drawback of the Quick-Core needle is that it is difficult to maneuver, especially for transduodenal biopsy. Recently, a new 22G needle with a reverse bevel at the tip (EUS-FNB) has become available. The ProCore needle design combines the features of an FNA needle with a core biopsy needle [20], which has 
enhanced flexibility, especially for core tissue collection. Results from a multicenter study on EUS-FNB showed that, overall, the operations were completed in $98.24 \%$ patients, the histological specimens were adequate in $89.47 \%$ patients, and the diagnoses were accurate in $92.9 \%$ patients [11]. EUS-guided tissue acquisition now seems to be more suitable for histological evaluation $[13,20]$. Here, we carry out a randomized controlled trial to compare the diagnostic efficiency of EUS-FNB versus EUS-FNA and to assess the sample quality obtained by different types of needles with different methods. To our knowledge, this is the largest cohort study on EUS-FNB so far. The outcome measurements will help us to understand the special indications for each technique and their diagnostic accuracy. In addition, Aadam et al. reported a significant rescue effect of FNA crossover to FNB [13]. We specifically designed our trial to allow the patient to crossover to the other arm if diagnosis is not achieved after the first round of needle biopsy, which will help us draw our conclusions.

The success of EUS-FNA is associated with several factors, including appropriate methods of biopsy, sample preparation, and onsite cytopathological interpretation [31]. The onsite cytopathological interpretation may not always be available during EUS-FNA procedures; therefore, appropriate methods of biopsy and sample preparation are very important. Some echoendoscopists use slow-pull, and others use constant suction. Suction with a self-retracting syringe will likely bring more cellularity but also more blood. In this trial, the echoendoscopist will use both methods (slow-pull and constant suction) on each lesion and, hopefully, will be able to draw a clear conclusion on selecting which biopsy method should be used for which procedure. Furthermore, standard procedures and sample preparation methods will be used to process all types of biopsy samples. For example, after the needle has been inserted into the mass, for both FNA and FNB, the needle will be moved back and forth 20 times within the lesion to collect specimens. The specimens will be expelled from a needle in three steps: step 1, which involves pushing the stylet into the needle; step 2, which involves flushing the needle with $0.1 \mathrm{ml}$ saline; and step 3, which involves filling the needle with $2 \mathrm{ml}$ of air.

Some limitations exist in the present study design. For instance, we will not be performing the rapid onsite evaluation (ROSE) in the current trial. ROSE is most frequently used in the United States, and the absence of an onsite cytopathologist has been shown to affect the diagnostic yield by increasing the number of inadequate samples. Although its popularity is growing here in China as well, several alternative strategies, including the macroscopic onsite evaluation (MOSE), have been reported to improve the diagnostic yield [21]. The well- trained endoscopists in our centers will apply MOSE and gross inspection to evaluate the quality of the specimens. Second, although the crossover design is critical, obtaining agreement from the patients for the second biopsy may be difficult because it may increase the risk of complications. Consequently, the number of patients recruited may be reduced, thereby leading to suboptimal conclusions. Third, some patients may not undergo surgery and, hence, will not have any final pathology report. In addition, the possibility exists that contact with the patient will be lost during the 48 weeks of follow-up. To prevent dropout, local research assistants will be recruited to help complete the patient registration information (record at least two phone numbers) and ensure that appointments are being made for followup assessments.

\section{Trial status}

Patient enrollment began on December 20, 2014 and completion is expected by December 31, 2015. At present (December 5, 2015), 372 patients have been enrolled in the study.

\section{Abbreviations \\ AFP: Alpha fetal protein; ALP: Alkaline phosphatase; ALT: Alanine aminotransferase; APTT: Activated partial thromboplastin time; AST: Aspartate aminotransferase; BUN: Blood urea nitrogen; CA19-9: Carbohydrate antigen 19-9; CA72- 4: Carbohydrate antigen72-4; CEA: Carcinoembryonic antigen; Cr: Creatinine; EUS: Endoscopic ultrasonography; EUS-FNA: Endoscopic ultrasound-guided fine-needle aspiration; EUS-FNB: Endoscopic ultrasound-guided fine-needle biopsy; FNA: Fine needle aspiration; INR: International normalized ratio; MOSE: Macroscopic onsite evaluation; NPV: Negative predictive value; NSE: Neuron specific enolase; PPV: Positive predictive value; PT: Prothrombin time; ROSE: Rapid onsite evaluation: \\ SA: Surface area; SAS: Statistical Analysis System; SSC: Squamous cell carcinoma antigen; TT: Thrombin time.}

\section{Competing interests}

The authors declare that they have no competing interests.

\section{Authors' contributions}

JLW and BC conceived the study and prepared a draft manuscript. YWL, $\mathrm{XLW}$, and YW participated in the design of the intervention and the development of the recruitment strategy. WH and QZG participated in the design of the intervention. PY participated in the design of the randomization system and advised on the sample size calculation. All authors contributed to the study design and commented on the manuscript. All authors read and approved the final manuscript.

\section{Acknowledgements}

We acknowledge Prof. Guiqi Wang and Prof. Xiujiang Yang for their advice for this study protocol. We would like to thank colleagues from the Tongji Hospital, Tongji Medical College, Huazhong University of Science and Technology, Cancer Hospital Chinese Academy of Medical Sciences, Fudan University Shanghai Cancer Center, Sun Yat-Sen University Cancer Center, and The First affiliated Hospital of Zhejiang University for their efforts in the project implementation. The study is an investigator-initiated trial and did not receive any extra funding.

\section{Author details}

'Department of Gastroenterology and Hepatology, Tongji Hospital, Tongji Medical College, Huazhong University of Science and Technology, Wuhan 430030, China. ${ }^{2}$ School of Public Health, Tongji Medical College, Huazhong University of Science and Technology, Wuhan 430030, China. 
Received: 6 December 2015 Accepted: 31 March 2016

\section{Published online: 12 April 2016}

\section{References}

1. Strand DS, Jeffus SK, Sauer BG, Wang AY, Stelow EB, Shami VM. EUS-guided 22-gauge fine-needle aspiration versus core biopsy needle in the evaluation of solid pancreatic neoplasms. Diagn Cytopathol. 2014;42:751-8.

2. Weston BR, MAMS. Optimizing diagnostic yield for eus-guided sampling of solid pancreatic lesions: a technical review. Gastroenterol Hepatol. 2013;9: 352-63.

3. Mehmood S, Loya A, Yusuf MA. Clinical utility of endoscopic ultrasoundguided fine-needle aspiration in the diagnosis of mediastinal and intraabdominal lymphadenopathy. Acta Cytol. 2013;57:436-42.

4. DeWitt J, Jowell P, Leblanc J, McHenry L, McGreevy K, Cramer H, et al. EUSguided FNA of pancreatic metastases: a multicenter experience. Gastrointest Endosc. 2005;61:689-96.

5. Puli SR, Batapati KRJ, Bechtold ML, Ibdah JA, Antillon D, Singh S, et al. Endoscopic ultrasound: it's accuracy in evaluating mediastinal lymphadenopathy? A meta-analysis and systematic review. World J Gastroenterol. 2008;14:3028-37.

6. Puri R, Vilmann P, Sud R, Kumar M, Taneja S, Verma K, et al. Endoscopic ultrasound-guided fine-needle aspiration cytology in the evaluation of suspected tuberculosis in patients with isolated mediastinal lymphadenopathy. Endoscopy. 2010;42:462-7.

7. Savides TJ, Donohue M, Hunt G, Al-Haddad M, Aslanian H, Ben-Menachem T, et al. EUS-guided FNA diagnostic yield of malignancy in solid pancreatic masses: a benchmark for quality performance measurement. Gastrointest Endosc. 2007:66:277-82

8. Sadeghi A, Mohamadnejad M, Islami F, Keshtkar A, Biglari M, Malekzadeh R, et al. Diagnostic yield of EUS-guided FNA for malignant biliary stricture: a systematic review and meta-analysis. Gastrointest Endosc. 2016;83:290-8.

9. Lee JK, Choi JH, Lee KH, Kim KM, Shin JU, Lee JK, et al. A prospective, comparative trial to optimize sampling techniques in EUS-guided FNA of solid pancreatic masses. Gastrointest Endosc. 2013;77:745-51.

10. Ogura T, Yamao K, Sawaki A, Mizuno N, Hara K, Hijioka S, et al. Clinical impact of K-ras mutation analysis in EUS-guided FNA specimens from pancreatic masses. Gastrointest Endosc. 2012;75:769-74.

11. Iglesias-Garcia J, Poley J, Larghi A, Giovannini M, Petrone MC, Abdulkader I, et al. Feasibility and yield of a new EUS histology needle: results from a multicenter, pooled, cohort study. Gastrointest Endosc. 2011;73:1189-96.

12. Van Riet PA, Cahen DL, Poley J, Bruno MJ. A global survey evaluating the global practice patterns of EUS-guided tissue sampling; preferences for tissue acquisition and processing [abstract]. Gastrointest Endosc. 2015;81: AB430-1.

13. Aadam AA, Amick AE, Shah JN, Hamerski CM, Bhat YM, Klapman JB, et al. A multicenter prospective randomized controlled cross-over trial comparing endoscopic ultrasound (EUS)-guided fine needle aspiration (FNA) and fine needle biopsy (FNB) for pancreatic and non-pancreatic masses [abstract]. Gastrointest Endosc. 2014;79:AB188-9.

14. Ashida R, Yasukawa S, Yanagisawa A, Kamata K, Kudo M, Ogura T, et al. Prospective multicenter randomized controlled trial of histological diagnostic yield comparing 25G EUS-FNA needles with and without a core trap in patients with solid pancreatic masses [abstract]. Gastrointest Endosc. 2014;79:AB111.

15. Choi HJ, Moon JH, Kim HK, Lee YN, Kim DC, Lee TH, et al. Comparison of EUS-fine needle biopsy with EUS-fine needle aspiration as a historical control for diagnosis of pancreatic solid masses [abstract]. Gastrointest Endosc. 2013;77:AB401.

16. Alatawi A, Beuvon F, Grabar S, Sarah L, Chaussade S, Terris B, et al. Comparison of fenestrated versus standard needles for endoscopic ultrasound-guided biopsy of solid pancreatic lesions [abstract]. Gastrointest Endosc. 2014;79:AB428-9.

17. Witt BL, Adler DG, Hilden K, Layfield LJ. A comparative needle study: EUSFNA procedures using the HD ProCore ${ }^{\mathrm{TM}}$ and EchoTip ${ }^{\oplus}$ 22-gauge needle types. Diagn Cytopathol. 2013;41:1069-74.

18. Alatawi A, Beuvon F, Grabar S, Leblanc S, Chaussade S, Terris B, et al. Comparison of $22 \mathrm{G}$ reverse-beveled versus standard needle for endoscopic ultrasound-guided sampling of solid pancreatic lesions. United Eur Gastroenterol J. 2015;3:343-52.

19. Nagula S, Pourmand K, Aslanian HR, Bucobo JC, Dimaio CJ, Gonda TA, et al. EUS-fine needle aspiration (FNA) vs. EUS-fine needle biopsy (FNB) for solid mass lesions: interim analysis of a large multicenter, randomized clinical trial [abstract]. Gastrointest Endosc. 2013;77:AB357-8.

20. Iwashita T, Nakai Y, Samarasena JB, Park DH, Zhang Z, Gu M, et al. High single-pass diagnostic yield of a new 25-gauge core biopsy needle for EUSguided FNA biopsy in solid pancreatic lesions. Gastrointest Endosc. 2013;77: 909-15.

21. Iwashita T, Yasuda I, Mukai T, Doi S, Nakashima M, Uemura S, et al. Macroscopic on-site quality evaluation of biopsy specimens to improve the diagnostic accuracy during EUS-guided FNA using a 19-gauge needle for solid lesions: a single-center prospective pilot study (MOSE study). Gastrointest Endosc. 2015;81:177-85.

22. Fabbri C, Luigiano C, Maimone A, Tarantino I, Baccarini P, Fornelli A, et al. Endoscopic ultrasound-guided fine-needle biopsy of small solid pancreatic lesions using a 22-gauge needle with side fenestration. Surg Endosc. 2015; 29:1586-90.

23. Wani S, Early D, Kunkel J, Leathersich A, Hovis CE, Hollander TG, et al. Diagnostic yield of malignancy during EUS-guided FNA of solid lesions with and without a stylet: a prospective, single blind, randomized, controlled trial. Gastrointest Endosc. 2012;76:328-35.

24. Dwyer J, Pantanowitz L, Ohori NP, Pai RK, Vrbin C, Brand RE, Monaco SE, Endoscopic ultrasound-guided FNA and ProCore biopsy in sampling pancreatic and intra-abdominal masses. Cancer Cytopathol. 2016;124(2):11021. doi:10.1002/cncy.21623

25. Gleeson FC, Kipp BR, Caudill JL, Clain JE, Clayton AC, Halling KC, et al. False positive endoscopic ultrasound fine needle aspiration cytology: incidence and risk factors. Gut. 2010;59:586-93.

26. Hawes $\mathrm{RH}$. The evolution of endoscopic ultrasound: improved imaging, higher accuracy for fine needle aspiration and the reality of endoscopic ultrasound-guided interventions. Curr Opin Gastroenterol. 2011;26:1.

27. Navina S, McGrath K, Chennat J, Singh V, Pal T, Zeh H, et al. Adequacy assessment of endoscopic ultrasound-guided, fine-needle aspirations of pancreatic masses for theranostic studies: optimization of current practices is warranted. Arch Pathol Lab Med. 2014:138:923-8.

28. Song TJ, Kim JH, Lee SS, Eum JB, Moon SH, Park DH, et al. The prospective randomized, controlled trial of endoscopic ultrasound-guided fine-needle aspiration using 22G and 19G aspiration needles for solid pancreatic or peripancreatic masses. Am J Gastroenterol. 2010;105:1739-45.

29. Ginès A, Wiersema MJ, Clain JE, Pochron NL, Rajan E, Levy MJ. Prospective study of a Trucut needle for performing EUS-guided biopsy with EUSguided FNA rescue. Gastrointest Endosc. 2005;62:597-601.

30. Thomas T, Kaye PV, Ragunath K, Aithal G. Efficacy, safety, and predictive factors for a positive yield of EUS-guided Trucut biopsy: a large tertiary referral center experience. Am J Gastroenterol. 2009;104:584-91.

31. Savides TJ. Tricks for improving EUS-FNA accuracy and maximizing cellular yield. Gastrointest Endosc. 2009;69:S130-3.

\section{Submit your next manuscript to BioMed Central and we will help you at every step:}

- We accept pre-submission inquiries

- Our selector tool helps you to find the most relevant journal

- We provide round the clock customer support

- Convenient online submission

- Thorough peer review

- Inclusion in PubMed and all major indexing services

- Maximum visibility for your research

Submit your manuscript at www.biomedcentral.com/submit 